\title{
Multivariable Control Strategy for a refrigeration plant
}

\author{
Liset Mayo Martí ${ }^{1}$, Miguel Alejandro Botello Lemus ${ }^{2}$
}

\author{
1,2, Grupo de Automatización, Acinox Las Tunas, Circunvalante Norte Km 3 1², Zona Industrial, Las Tunas, Cuba. \\ Email: yinef@ind.cujae.edu.cu,dianelysra@ind.cujae.edu.cu,abel@ind.cujae.edu.cu,marthagom@tesla.cujae.edu.cu
}

Received: December 19 $1{ }^{\text {th }}, 2017$.

Accepted: January $09^{\text {th }}, 2018$.

Published: March 30 ${ }^{\text {th }}, 2018$.

Copyright (C2016 by authors and Institute of Technology Galileo of Amazon (ITEGAM).

This work is licensed under the Creative Commons Attribution International

License (CC BY 4.0).

http://creativecommons.org/licenses/by/4.0/ (c) (1) (3) Open Accest

\begin{abstract}
In this paper a multivariable control system for the heat exchanger of a real plant is proposed; using pi direct synthesis controllers and simplified type decouplers. In addition, the measured, controlled and manipulated variables of the units that constitute this plant and that interact are analyzed, so as not to cause conflicts between them. The cascade control configuration is chosen for the control of air temperature, which is the main function of the study plant. The results obtained at simulation scale demonstrated the effectiveness of the proposal.
\end{abstract}

Keywords: Multivariable systems, multivariable control, plantwide control, steel industry, refrigeration plant.

\section{Estrategia de Control Multivariable para una planta frigorífica}

\section{RESUMEN}

En este trabajo se propone un sistema de control multivariable para el intercambiador de calor de una planta real; usando controladores PI de Síntesis Directa y desacopladores de tipo Simplificado. Además, se analizan las variables medidas, controladas y manipuladas, de unidades que constituyen dicha planta y que interactúan, para no provocar conflictos entre estas. Se elige la configuración de control cascada para el control de la temperatura del aire, que es la función principal de la planta objeto de estudio. Los resultados obtenidos a escala de simulación demostraron la efectividad de la propuesta.

Palabras Claves: Sistemas Multivariables, Control Multivariable, Control de Plantas Completas, Industria del acero, Grupo Frigorífico.

\section{INTRODUCCIÓN}

El control multivariable constituye un tópico de avanzada en el campo del control de procesos. Desarrollado originalmente para resolver problemas específicos de control en refinerías, la tecnología de control multivariable se utiliza hoy en día en plantas químicas, sector alimentario, automóvil, metalurgia, industria papelera y otros entornos industriales. Es una tecnología consolidada y utilizada para sistemas de control de plantas completas.

Los sistemas multivariables, por definición, son sistemas con varias entradas y salidas, en los que una entrada afecta a varias salidas y recíprocamente una salida es afectada por varias entradas. La interacción entre sus variables representa un fenómeno único de los sistemas multivariables, lo cual puede dificultar en gran medida el diseño del sistema de control [1].

Tradicionalmente los problemas de control de procesos se han abordado mediante lazos de control monovariables e independientes, donde una variable de medida es controlada con una variable manipulada. En dichos lazos, el controlador proporcional-integral-derivativo (PID) es el más extendido, ya que se puede entender e implementar fácilmente, y se logra integrar en configuraciones de control cascada, feedforward, etc. Estos enfoques se han desarrollado a lo largo de varios años de experiencia, y funcionan adecuadamente cuando las interacciones entre las diferentes variables del proceso son pequeñas. Sin embargo, cuando las interacciones son importantes, el control de un sistema multivariable se puede complejizar. Por ejemplo, el ajuste de uno de los lazos puede afectar considerablemente al rendimiento de otros, hasta el punto incluso de desestabilizarlos [2]. En estos casos, los tradicionales y bien asentados métodos de sintonía monovariable no consiguen una respuesta del sistema satisfactoria, y a menudo los controladores se ajustan de manera ineficiente. Ello justifica el interés por el desarrollo de metodologías de diseño de control multivariable que tengan en cuenta el problema de la interacción. 
Una filosofía de diseño es desacoplar el sistema, con la intención de eliminar o reducir las interacciones entre las variables del proceso. De esta forma, el sistema multivariable se descompondría en varios sistemas monovariables donde no habría acoplamientos entre las variables de referencia deseadas y las salidas controladas de la planta. Hay que decir que el control por desacoplo no es solo popular porque simplifique el sistema multivariable, sino porque además, es una característica deseada en muchas aplicaciones prácticas. Primero, el desacoplamiento es necesario para facilitar la labor de los técnicos, al menos en la industria química y de procesos, ya que de lo contrario, sería muy difícil decidir los múltiples valores de referencia del sistema multivariable para alcanzar los objetivos finales. Segundo, un buen desacoplamiento puede ayudar a mejorar la respuesta en lazo cerrado. Algunos importantes fabricantes de controladores, como Yokogawa o Foxboro, consideran el mal desacoplamiento como uno de los principales problemas en la industria.

En algunos procesos con poca o mediana interacción, los acoplamientos se pueden reducir aceptablemente con un control descentralizado, donde por cada lazo hay un controlador que trabaja independientemente. Sin embargo, en procesos con una interacción severa es necesario acudir a técnicas de mayor complejidad.

Para ello, en el ámbito del diseño de sistemas de control lineales podemos encontrar diversos planteamientos, desde el enfoque tradicional basado en matrices de funciones de transferencia hasta otras metodologías como el control lineal cuadrático gaussiano (LQG), el posterior control óptimo $\mathrm{H} \infty$, el control por modelo interno (IMC) o el control predictivo basado en modelos (MPC), que ya se aplican en algunos sistemas de control industriales [2].

La presente propuesta se basa en aplicar las metodologías de diseño de control multivariable a un proceso real, de la industria siderúrgica, donde el control se realiza de manera ineficiente provocando menores beneficios económicos. Se trata del grupo frigorífico de la planta de fraccionamiento de aire de la industria de aceros Acinox Las Tunas. Constituyen al mismo varias unidades interconectadas; de ellas, un intercambiador de calor, de naturaleza esencialmente multivariable.

A partir de lo antes descrito, este artículo tiene como objetivo proponer un sistema de control multivariable lineal con desacoples, usando controladores de Síntesis Directa para un intercambiador de calor; y mediante configuración de control cascada regular la temperatura de salida del aire, que es la función principal de la planta objeto de estudio. El artículo se organizó como sigue: en la sección dos se describe el proceso del grupo frigorífico, en la sección tres se exponen las bases y la formulación del problema, en la sección cuatro se presenta el resultado principal revelando su efectividad, en la sección 5 se plantean las conclusiones y en la seis las recomendaciones.

\section{FUNCIONAMIENTO DE LA PLANTA FRIGORÍFICA}

En la industria Acinox Las Tunas donde se fabrican aceros bajos al carbono en forma de palanquillas, la planta de Fraccionamiento de Aire se encarga de producir los gases oxígeno, nitrógeno y argón que posteriormente se insuflan en el acero líquido para lograr las reacciones químicas que garantizan un ajuste de la composición del mismo. En el proceso de tratamiento del aire interviene el llamado grupo frigorífico.

El grupo frigorífico tiene como objetivo enfriar el aire a una temperatura de $8^{\circ} \mathrm{C}$, que es determinada por la temperatura de evaporación y por la cantidad de fluido refrigerante introducido en el evaporador. El aire caliente que debe ser enfriado y desecado, entra en el evaporador donde es llevado a una temperatura inferior a su punto de rocío, mediante el intercambio de calor con el fluido refrigerante.

El circuito del fluido refrigerante está repartido en dos secciones principales: sección de alta presión y sección de baja presión. El compresor aspira el refrigerante en estado gaseoso, lo comprime y lo envía, siempre en estado gaseoso pero a temperatura elevada al condensador, a través de la válvula de impulsión y el conducto impelente.

En el condensador el refrigerante se licua, manteniendo constante su presión. El refrigerante líquido sale luego del condensador a través de la válvula del líquido y pasa al filtro deshidratador y luego al intercambiador; finalmente, a través de la válvula de solenoide, llega a la válvula de expansión, tal como se muestra en la Figura 1. Esta válvula marca el límite entre la sección de alta presión y la de baja presión del circuito, ya que obliga al fluido refrigerante a expandirse en el evaporador con una temperatura y una presión mucho más bajas.

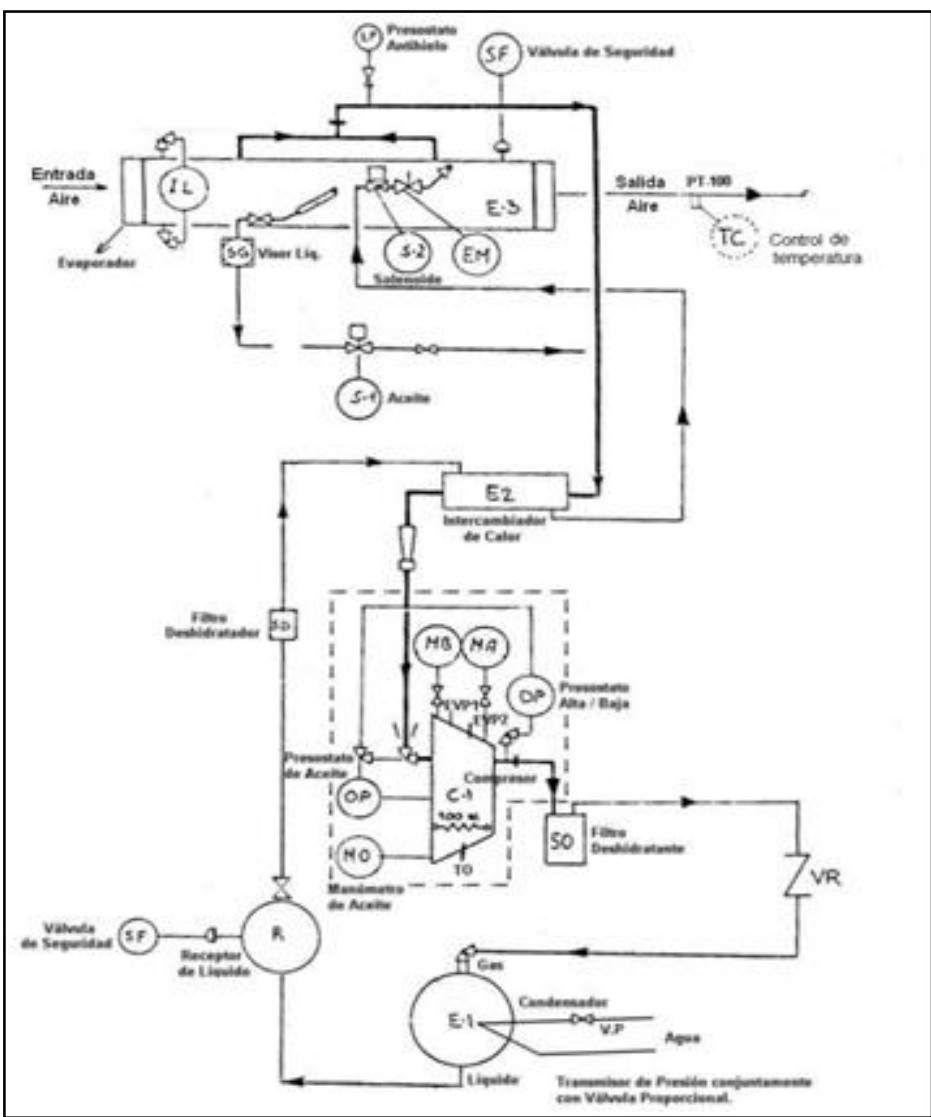

Figura 1: Proceso de enfriamiento del aire en el Grupo Frigorífico.

Fuente: Los autores, (2018).

En el evaporador el refrigerante pasa totalmente del estado líquido al estado gaseoso, substrayendo una determinada cantidad de calor del aire que circula en el evaporador mismo y provocando por lo tanto su enfriamiento. El gas que sale del evaporador pasa luego al intercambiador de calor, donde substrae calor al refrigerante.

En el Intercambiador de calor se sobrecalienta el gas aspirado por el compresor, haciendo evaporar eventuales partículas de líquido presentes en el gas que llega del evaporador. Además aumenta la capacidad de la instalación, por medio del subenfriamiento del líquido. Por lo que representa un punto importante para el máximo aprovechamiento de la instalación. 


\section{BASES Y FORMULACIÓN DEL PROBLEMA}

\section{III.1 SISTEMAS DE CONTROL MULTIVARIABLE}

Los sistemas de control multivariable son sistemas con varias entradas y salidas (MIMO), en los que una entrada afecta a varias salidas y recíprocamente una salida es afectada por varias entradas, como muestra la Figura 2, siendo el caso $2 \times 2$ el más tratado en la bibliografía [3-5], bien porque corresponde a procesos típicos reales, bien porque el proceso ha sido descompuesto en bloques de este tamaño [3][6].

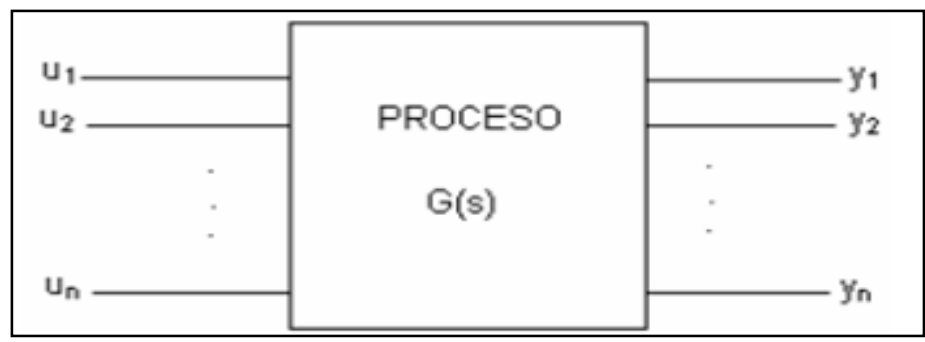

Figura 2: Proceso MIMO.

Fuente: Los autores, (2018).

El control de estos procesos, en la mayoría de los casos, es complejo y por lo general se deben tener en cuenta varios interrogantes para dar solución al problema de control [7], como: ¿cuál es la mejor agrupación por pares de variables controladas y manipuladas?, ¿cuánta interacción existe entre los diferentes lazos de control y como se afecta la estabilidad de los lazos?, ¿se puede hacer algo para reducir la interacción entre los lazos? y otras que trataremos brevemente a continuación.

\section{III.1.1 MEDIDAS DE INTERACCIÓN}

La dificultad de controlar un proceso multivariable no reside solo en el número de variables, sino más bien en la interacción que existe entre ellas. Este grado de interacción determinará si la estrategia de control a seleccionar para controlar el proceso será descentralizada o centralizada. Por lo tanto, es necesario disponer de medidas de interacción que ayuden a tomar dicha decisión. Desde que en [8] se presentara la matriz de ganancias relativas (RGA), ésta se ha convertido en una de las medidas más extendidas para cuantificar la interacción de un proceso multivariable. Si bien inicialmente solo implicaba consideraciones en estado estacionario, también se puede usar una RGA dinámica dependiente de la frecuencia. La RGA de una matriz compleja y no singular $\mathrm{G}$ es otra matriz compleja definida de la siguiente manera:

$$
\Lambda=G \times\left(G^{-1}\right)^{T}
$$

donde $\times$ denota el producto elemento a elemento (o producto Hadamard).

Aunque algunos autores opinan que la RGA es solo una técnica heurística sin una base teórica fuerte, se han establecido conexiones rigurosas entre la RGA y la estabilidad para sistemas con dos entradas y dos salidas (TITO) a partir de sus funciones de transferencia [9]. Sin entrar en mucho detalle y considerando la interpretación original de la RGA en estado estacionario [10], cada elemento $\lambda_{\mathrm{ij}}$ representa el efecto que sobre la ganancia del proceso entre la variable controlada $y_{i}$ y la variable manipulada $u_{j}$, tiene el hecho de cerrar todos los demás lazos de control (que deben ser estables y con integrador). Por tanto, el valor numérico de $\lambda_{\mathrm{ij}}$ es una medida del grado de interacción que los demás lazos de control tienen sobre el lazo de control que emparejaría $u_{j}$ con $\mathrm{y}_{\mathrm{i}}$. Si $\lambda_{\mathrm{ij}}=1$, la ganancia del proceso vista desde la entrada $\mathrm{u}_{\mathrm{j}}$ a la salida $y_{i}$ es la misma cuando todos los demás lazos de control están abiertos que cuando están cerrados. Esto indica que no existe interacción, al menos sobre el estado estacionario, entre el lazo analizado y los demás. Cuanto más se desvíe el valor $\lambda$ ij de la unidad, mayor será el grado de interacción de los lazos de control. Si la RGA fuera la matriz identidad no habría interacción.

\section{III.1.2 EMPAREJAMIENTO DE VARIABLES CONTROLADAS Y MANIPULADAS}

Debe tenerse en cuenta la siguiente serie de pasos para la selección del mejor par de variables controladas y manipuladas, seguidamente se presenta la secuencia de pasos comúnmente utilizada [11]:

1. Obtener la matriz de ganancias relativas, esta se calcula mediante la Ecuación (1).

2. Tener en cuenta algunos criterios de restricción como el no formar lazos de control con ganancias relativas negativas, ni con ganancia relativa infinita, ni con ganancia relativa nula.

3. Tener en cuenta algunos criterios de prioridad como son: controlar las variables más importantes con aquellas variables de entrada con las que tengan una respuesta dinámica más rápida sin respuesta inversa, cerrar lazos de control con las ganancias relativas más próximas a uno.

4. Tener en cuenta algunos criterios complementarios como son: la simulación de las distintas alternativas, emparejar variables con ganancia relativa ante perturbación baja, índice de estabilidad bajo.

\section{III.1.3 ANÁLISIS DE ESTABILIDAD}

Cuando se seleccionan apareamientos también resulta indispensable tomar en cuenta consideraciones de estabilidad entre los lazos. Dicha consideración se plantea en términos del siguiente teorema de estabilidad de Niederlinski [12].

El teorema establece que un sistema de control de lazos múltiples será inestable (para todos los posibles valores de los parámetros del controlador) si el índice de Niderlinski (N) definido como:

$$
N=\frac{\operatorname{det}[G(0)]}{\prod_{i=1}^{\mathrm{n}} g_{\mathrm{il}}(0)}
$$

es negativo.

El teorema de Niederlinski representa condiciones necesarias y suficientes únicamente para sistemas TITO. Para sistemas de orden mayor únicamente representa condiciones suficientes. Es decir, si el teorema se cumple, entonces el sistema de múltiples lazos será definitivamente inestable. Pero si el teorema no se cumple, el sistema de múltiples lazos puede ser o no inestable (en este caso, la estabilidad depende de los valores asignados a los parámetros del controlador).

\section{III.1.4 CONTROL POR DESACOPLO}

El control por desacoplo, básicamente se puede enfocar de dos formas, una de ellas es usando una red de desacoplo formada por los cuatro elementos $\operatorname{Dij}(\mathrm{s})$, con un controlador descentralizado diagonal. 
La Figura 3 muestra el esquema general de un sistema de control combinando red de desacoplo y controlador diagonal, donde $\mathrm{G}(\mathrm{s})$, $\mathrm{D}(\mathrm{s})$ y $\mathrm{C}(\mathrm{s})$ son las matrices de funciones de transferencia $\mathrm{n} \times \mathrm{n}$ del proceso, la red de desacoplo y el controlador diagonal, respectivamente. El producto del proceso original y la red de desacoplo conformaría el nuevo proceso aparente: $\mathrm{Q}(\mathrm{s})=$ $\mathrm{G}(\mathrm{s}) \cdot \mathrm{D}(\mathrm{s})$. El bloque compensador $\mathrm{D}(\mathrm{s})$, o red de desacoplamiento, se diseña con la intención de eliminar, o al menos reducir, las interacciones del proceso, de tal manera que el controlador descentralizado $\mathrm{C}(\mathrm{s})$ manipule las variables $\mathrm{v}_{\mathrm{i}}$ en lugar de las variables $\mathrm{u}_{\mathrm{i}}$. Con esta configuración se pretende que el controlador vea al nuevo proceso aparente $\mathrm{Q}(\mathrm{s})$ como un conjunto de $\mathrm{n}$ procesos totalmente independientes o con mucha menos interacción, para los cuales se diseñaría el control descentralizado $\mathrm{C}(\mathrm{s})$ mediante técnicas de ajuste monovariable bien establecidas [13-15]. El controlador multivariable resultante de este diseño estaría compuesto por el control diagonal y la red de desacoplo.

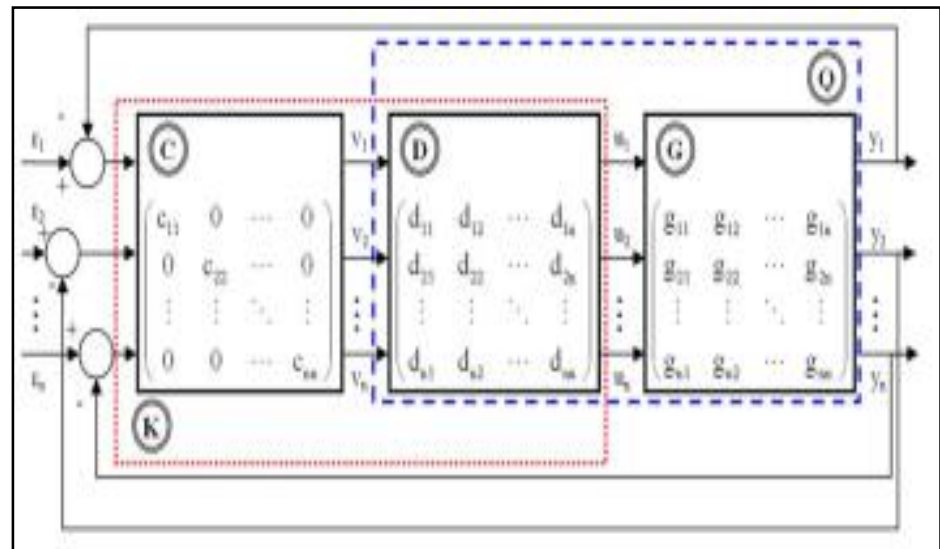

Figura 3: Sistema de control combinando red de desacoplamiento y controlador descentralizado.

Fuente: Los autores, (2018).

\section{III.2 SISTEMAS DE CONTROL PARA PLANTAS COMPLETAS}

El diseño de sistemas de control para plantas completas es la tarea más compleja para los ingenieros en instrumentación y control. Las dificultades se agravan por las fuertes no linealidades de los procesos, el conocimiento impreciso del mismo, su carácter multivariable y las interacciones entre variables [1].

Tradicionalmente, se supone que el diseño de un sistema de control para un planta tiene lugar después que una carta de flujo para el proceso haya sido sintetizada y diseñada, e incluso construida con detalles significativos. Esto permite conocer las unidades que constituyen la planta y sus dimensiones, cómo se interconectan estas unidades, el rango y las condiciones de operación, los posibles disturbios, los problemas pueden surgir durante la arrancada y parada de la planta. Tal información es sumamente útil para el diseño efectivo del sistema de control.

Cuando el diseño de la planta ha sido realizado, el ingeniero de control se ve forzado a trabajar con un sistema fuertemente especificado o determinado. Aun así, su tarea es diseñar un sistema de control que asegure satisfactoriamente la operación de la planta. Está claro que debe existir cierto grado de coordinación y cooperación entre los diseñadores del proceso y los del sistema de control. El diseño del sistema de control se torna simple cuando el proceso presenta pocas unidades interactuantes, principalmente con arquitectura serie y sin fuertes requerimientos operacionales.
Se han publicado muy pocos artículos sobre el control de plantas completas [16], y la mayoría de los métodos de control se desarrollan para procesos específicos [17-18]. En [19-20] se recomiendan 15 pasos para llevar a cabo el problema de diseño de control para procesos de varias unidades. Este enfoque se desarrolla suponiendo que no hay instrumentación ni control. Obviamente, muchos proyectos de control implican una renovación de los controles existentes. Si bien este enfoque fue desarrollado para procesos de varias unidades, también se puede aplicar a sistemas de unidad única.

\section{RESULTADOS}

El intercambiador del Grupo Frigorífico de Acinox Las Tunas, es de tipo tubo y carcasa y la transferencia de calor se realiza entre el freón líquido que llega a la entrada del mismo con una temperatura de $45^{\circ} \mathrm{C}$ y el freón gaseoso a $15^{\circ} \mathrm{C}$. La función de este intercambiador de calor es reducir la temperatura del líquido refrigerante a un valor deseado para que llegue más frío al evaporador con el fin de enfriar el aire, mientras que el gas que llega al intercambiador se sobrecalienta pare eliminar contenido líquido que quedaba en él y pase al compresor sin provocarle ninguna afectación mecánica. De esta manera se incrementa la eficiencia del sistema.

Las variables a controlar entonces son: la temperatura a la salida del intercambiador, del freón líquido $\mathrm{T}_{\mathrm{fl}} \mathrm{y}$ del freón gaseoso $\mathrm{T}_{\mathrm{fg}}$. Las variables manipuladas son el flujo de entrada al intercambiador, del freón líquido $\mathrm{F}_{\mathrm{fl}} \mathrm{y}$ del freón gaseoso $\mathrm{F}_{\mathrm{fg}}$, como se ilustra en la Figura 4.

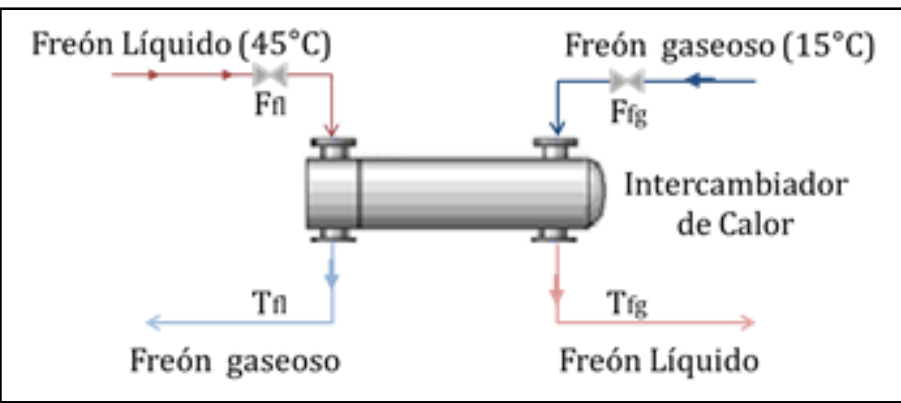

Figura 4: Intercambiador de Calor freón líquido- freón gaseoso da la planta objeto de estudio.

Fuente: Los autores, (2018).

El modelo matemático del intercambiador de calor fue tomado de [21] y se representa en el dominio de Laplace mediante las siguientes funciones de transferencia:

$$
\begin{gathered}
G_{11}(s)=\frac{0.4241}{148.6 s+1} \\
G_{12}(s)=\frac{-0.214}{(142.2 s+1)(28.4 s+1)} \\
G_{21}(s)=\frac{0.0695}{(126 s+1)(26.4 s+1)} \\
G_{22}(s)=\frac{-0.5}{150 s+1}
\end{gathered}
$$

Donde, $\mathrm{G}_{11}$ (s) es la función de transferencia que relaciona el flujo de freón líquido de entrada, con la temperatura del freón líquido de salida, $\mathrm{G}_{21}(\mathrm{~s})$ es la función de transferencia que relaciona el flujo de freón líquido de entrada, con la temperatura 
del freón gaseoso a la salida, $\mathrm{G}_{12}$ (s) es la función de transferencia que relaciona el flujo de freón gaseoso de entrada, con la temperatura del freón líquido de salida, $\mathrm{G}_{22}(\mathrm{~s})$ es la función de transferencia que relaciona flujo de freón gaseoso de entrada, con la temperatura del freón gaseoso a la salida.

\section{IV.1 ANÁLISIS DE LA INTERACCIÓN}

La primera pregunta que se debe hacer después de tener la caracterización dinámica del proceso, es saber si el apareamiento de las variables controladas y las manipuladas es el adecuado. Para este sistema solo fueron posibles dos combinaciones diferentes de las variables controladas con las variables manipuladas.

Para empezar, tiene sentido que cada variable controlada se controle mediante la variable manipulada que ejerce mayor "influencia" sobre aquélla. En este contexto, la influencia y la ganancia tienen el mismo significado $\mathrm{y}$, en consecuencia, para tomar una decisión se deben encontrar todas las ganancias del proceso. Específicamente, la matriz de ganancias de estado estacionario (MGEE) de lazo abierto del intercambiador de calor objeto de estudio es la siguiente:

$$
K=\left[\begin{array}{cc}
0.4241 & -0.214 \\
0.0695 & -0.5
\end{array}\right]
$$

Para normalizar la MGEE y hacerla independiente de las unidades, se utilizó la técnica que desarrolló Bristol. La matriz de ganancia relativa obtenida partir de la MGEE y basado en álgebra de matrices es:

$$
\Lambda=\left[\begin{array}{cc}
1.075 & -0.075 \\
-0.075 & 1.075
\end{array}\right]
$$

Cada elemento de la RGA se determinó multiplicando cada elemento de la matriz MGEE por su correspondiente en la matriz MGEE inversa. El cálculo de la matriz MGEE inversa es:

$K^{-1}=\frac{\operatorname{Adj}(K)}{\operatorname{Det}(K)}=\frac{\left[\begin{array}{cc}-0.5 & 0.214 \\ -0.0695 & 0.4241\end{array}\right]}{-0.197}=\left[\begin{array}{cc}2.538 & 0.3527 \\ -1.086 & -2.1527\end{array}\right]$

Entonces,

$\Lambda=\left[\begin{array}{cc}0.4241 & -0.214 \\ 0.0695 & -0.5\end{array}\right] *\left[\begin{array}{cc}2.538 & 0.3527 \\ -1.086 & -2.1527\end{array}\right]=\left[\begin{array}{cc}1.075 & -0.075 \\ -0.075 & 1.075\end{array}\right]$

En este caso se observan valores negativos en dicha matriz que denotan la peor interacción y por tanto inestabilidad en el lazo de control. En fin, la mejor pareja variable controlada-variable manipulada es la temperatura de salida del freón líquido con el flujo de entrada de freón líquido, dado por el modelo $\mathrm{G}_{11}(\mathrm{~s})$ de la ecuación 3, y la temperatura de salida del freón gaseoso con el flujo de entrada de freón gaseoso, representado por el modelo $\mathrm{G}_{22}$ (s) en la expresión 6. Así podemos decir que la usaremos la alternativa de acoplamiento 1-1/2-2, como se representa en la Figura 5.

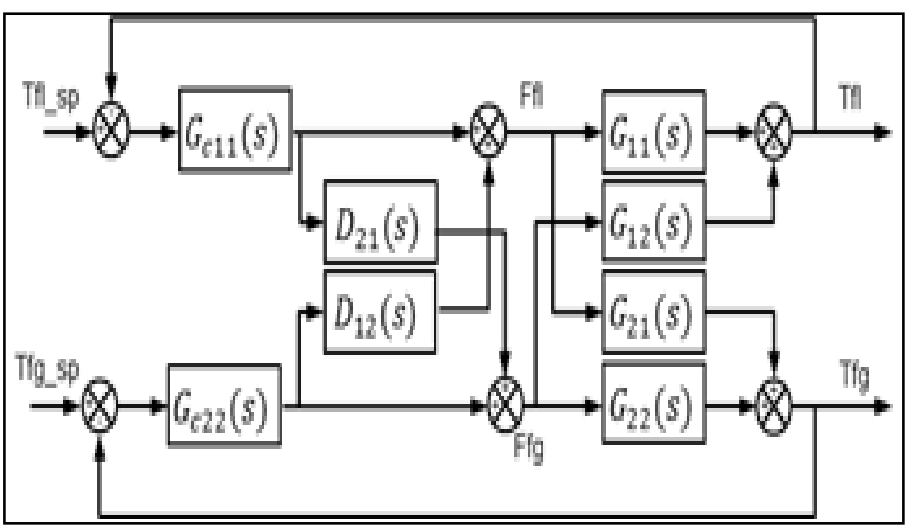

Figura 5: Diagrama en bloque del sistema de control para el Intercambiador de Calor.

Fuente: Los autores, (2018).

\section{IV.2 ANÁLISIS DE LA ESTABILIDAD}

El índice de Niederlinski, para el intercambiador caso de estudio, con el par de variables seleccionado se calculó de acuerdo a (2), resultando:

$$
N=\frac{|K|}{\prod_{i=1}^{2} K_{i j}}=\frac{-0.197}{0.4241 *(-0.5)}=0.929
$$

Es evidente el valor positivo del resultado, por tanto la selección del par de variables es el adecuado para diseñar una estrategia de control multivariable.

\section{IV.3 SINTONIZACIÓN DE LOS CONTROLADORES}

Para controlar las temperaturas de salida del freón líquido y gaseoso fueron ajustados los controladores PI, $\mathrm{G}_{\mathrm{C} 11}(\mathrm{~s})$ y $\mathrm{G}_{\mathrm{C} 22}(\mathrm{~s})$, mediante la estrategia de control de Síntesis Directa (1).

La función de transferencia de los controladores PI se expresan de la manera siguiente:

$$
G_{c 11}(s)=2.357 * \frac{149.6 s+1}{148.6 s}
$$

$$
G_{c 22}(s)=-2 * \frac{1500+1}{150 s}
$$

\section{IV.4 SÍNTESIS DE DESACOPLADORES}

Hasta el momento se ha demostrado que, aunque acoplemos las variables de acuerdo a las exigencias del arreglo de Bristol, las interacciones pueden persistir en alguna medida. El desacoplador simplificado sirve para reducir la interacción presente en el sistema. En la Figura 5 se presenta el diagrama de bloques del sistema de control con los desacopladores $\mathrm{D}_{\mathrm{ij}}$.

Para compensar la interacción del lazo de control de temperatura del freón gaseoso sobre el de temperatura de freón líquido se introduce el desacoplador $\mathrm{D}_{21}$ dado por:

$D_{21}(s)=-\frac{G_{21}}{G_{22}}=-\frac{\frac{0.0695}{(126 s+1)(26.4 s+1)}}{\frac{-0.5}{150 s+1}}=\frac{0.0139(150 s+1)}{(126 s+1)(26.4 s+1)}$

Y para compensar la interacción del lazo de control de temperatura del freón líquido sobre el de temperatura de freón gaseoso se introduce el desacoplador $\mathrm{D}_{12}$ dado por: 
$D_{12}(s)=-\frac{G_{12}}{G_{12}}=-\frac{\frac{-0.214}{(142.2 s+1)(28,4 s+1)}}{\frac{0.4241}{148.6 s+1}}=\frac{0.5045(148.6 s+1)}{(142.2 s+1)(28.4 s+1)}$

Ahora el sistema multivariable se comporta como dos sistemas de una entrada y una salida independientes.

\section{IV.5 SIMULACIÓN DEL SISTEMA DE CONTROL MULTIVARIABLE PROPUESTO}

Los resultados de simulación se obtuvieron mediante la herramienta Simulink del software Matlab®. Se realizaron varias pruebas con el fin de mostrar el comportamiento del sistema, respuesta a lazo abierto y cuando se aplican los controladores PI con y sin desacopladores.

Primero, observemos en la Figura 6 cómo influyen las variables manipuladas, flujo de freón líquido y gaseoso, sobre las temperaturas de salida del freón líquido y gaseoso.

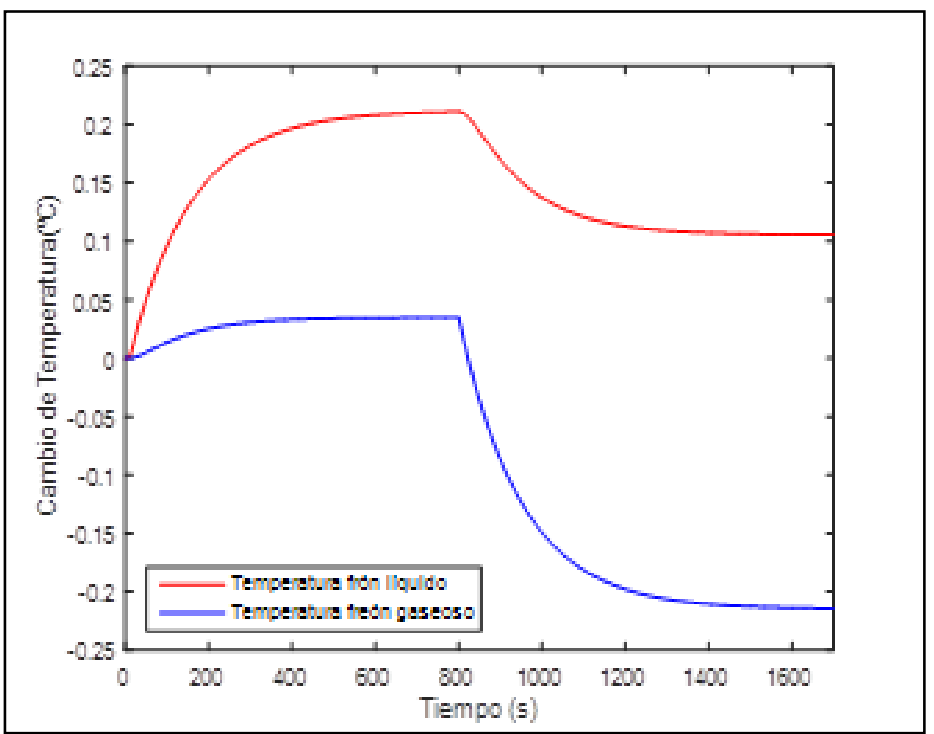

Figura 6: Respuesta del sistema multivariable a lazo abierto.

Fuente: Los autores, (2018).

Esta respuesta se obtuvo ante un cambio escalón de magnitud 0.5 en el flujo de entrada del freón líquido a los 10 segundos de haber iniciado la simulación; y posteriormente un cambio del flujo de entrada del freón gaseoso con igual magnitud, pero a los 800 segundos. Se aprecia claramente que cada una de las variables manipuladas, afecta a ambas salidas, por tanto estamos en presencia de un sistema multivariable completo.

Ahora veamos la respuesta del sistema al introducir los controladores determinados por las expresiones 9 y 10, mostrada en la Figura 7. Para esta prueba se sometió el sistema MIMO a un cambio en la referencia de temperatura del freón líquido (Tfl_sp) de $-3^{\circ} \mathrm{C}$ a los 10 segundos de iniciada la corrida, mientras que se realizó un cambio de $3^{\circ} \mathrm{C}$ en la señal de referencia de la temperatura del freón gaseoso a los 1000 segundos. Ambas señales de salida alcanzan el valor deseado sin ninguna oscilación ni sobrepasos, aunque presentan un tiempo de establecimiento un poco lento, debido a la dinámica del proceso. Puede observarse que aún existen interacciones en el proceso; sin embargo estas son eliminadas totalmente cuando incluimos al sistema de control los desacopladores calculados en la sección $4.4, \mathrm{D}_{21}$ y $\mathrm{D}_{12}$, como se aprecia en la Figura 8.

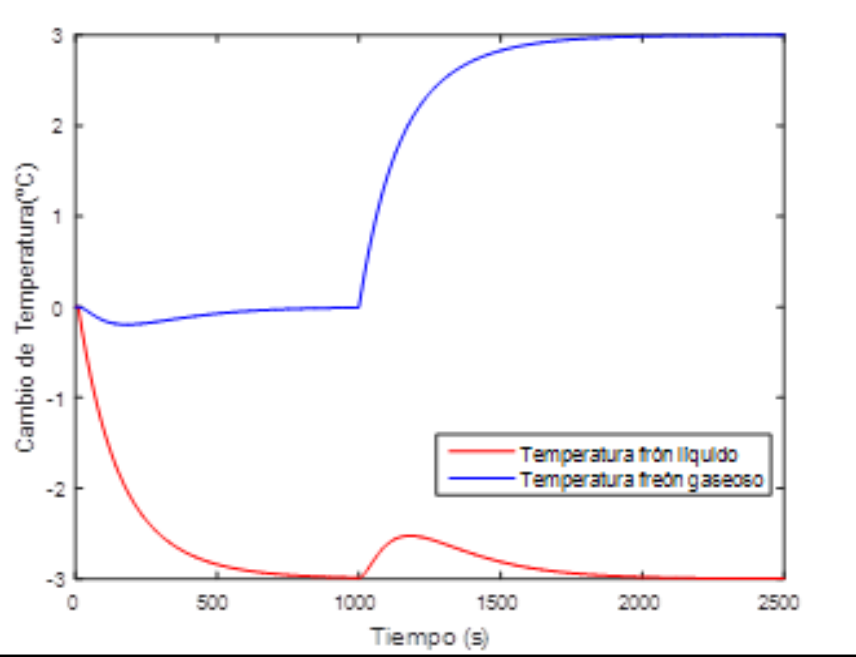

Figura 7: Respuesta del sistema multivariable con controladores de Síntesis Directa.

Fuente: Los autores, 2018.

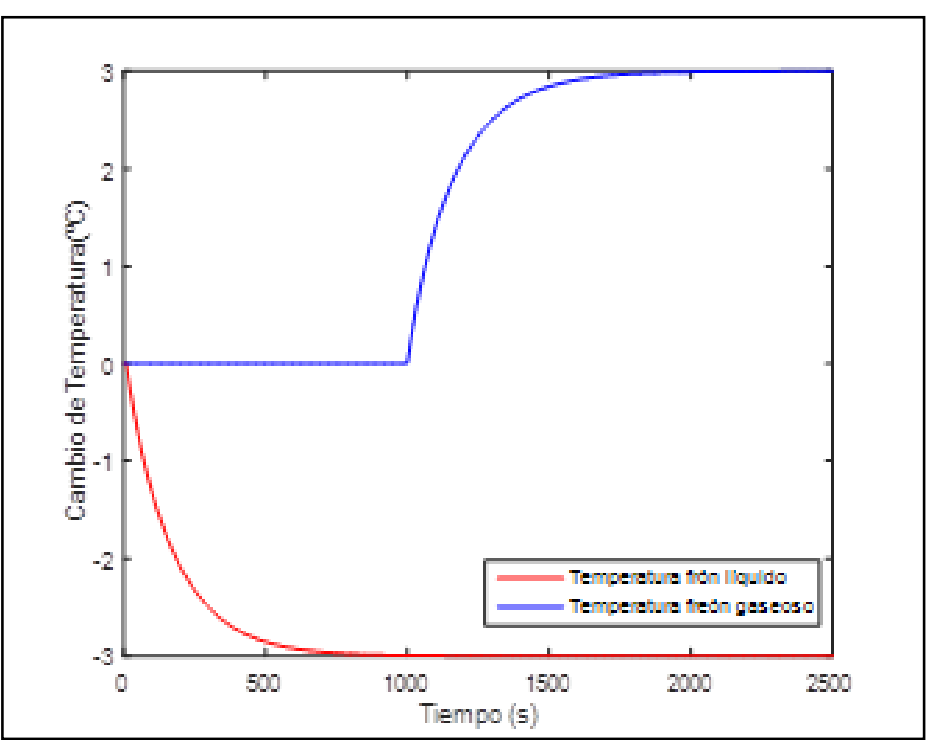

Figura 8: Respuesta del sistema multivariable con controladores de Síntesis Directa y desacopladores.

Fuente: Los autores, (2018).

\section{IV.6 CONEXIÓN DEL INTECAMBIADOR DE CALOR Y EL EVAPORADOR}

El evaporador del Grupo Frigorífico estudiado, tiene la función de enfriar el aire a una temperatura de $8^{\circ} \mathrm{C}$. Para ello se requiere regular el nivel del líquido refrigerante en el recipiente. El refrigerante, el freón líquido que viene desde el intercambiador analizado anteriormente, intercambia calor con el aire que entra al evaporador. Este proceso conlleva a la formación de vapor de freón, lo que ciertamente representa una perturbación de este sistema. La variable principal a controlar es la temperatura de salida del aire, a partir de regular el nivel del líquido refrigerante, manipulando el flujo de entrada del freón líquido en el evaporador. Siendo así, proponemos un sistema de control con configuración de control cascada; el lazo interno es el encargado de controlar el nivel del líquido refrigerante en el interior del evaporador, y el lazo de control externo controla entonces la temperatura del aire. De esta manera se impide algún conflicto entre el evaporador y el intercambiador de calor, como se muestra en la Figura 9. 


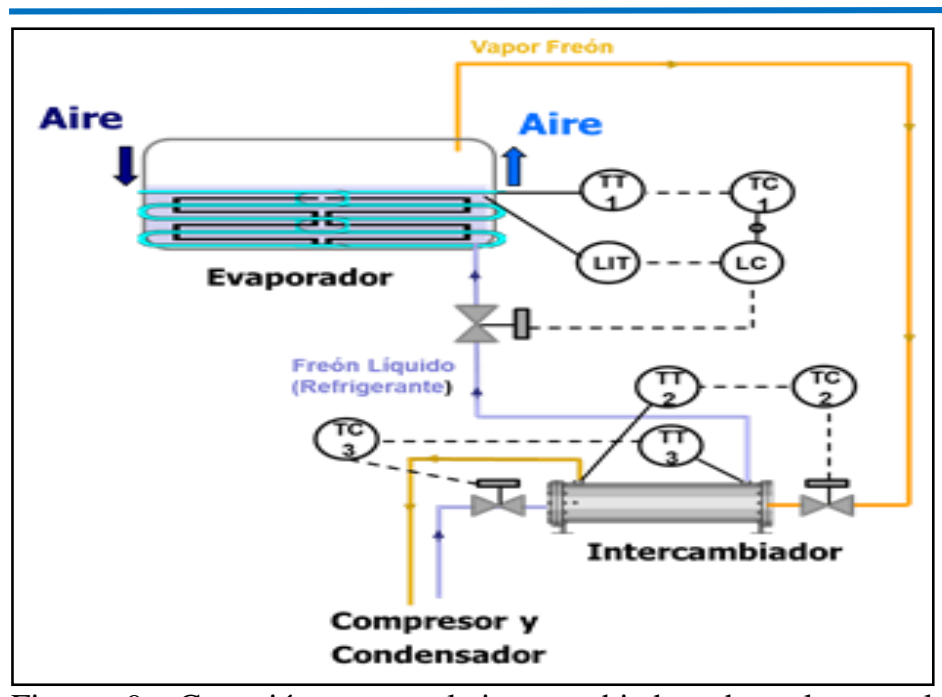

Figura 9: Conexión entre el intercambiador de calor y el evaporador.

Fuente: Los autores, (2018).

\section{CONCLUSIONES}

En este trabajo se propone un sistema de control para una planta real de la empresa Acinox Las Tunas. Se diseñó un sistema de control multivariable para el intercambiador de calor, usando controladores PI de síntesis directa y desacopladores simplificados que eliminaron completamente la interacción entre las variables. Se propuso además, una configuración de control cascada para la regulación de la temperatura en el evaporador, definiendo las variables controladas y manipuladas; respetando las consideraciones debidas para el trato de plantas completas que impiden conflictos entre sus unidades.

\section{RECOMENDACIONES}

Continuar profundizando en el estudio de los sistemas multivariable para definir una estrategia de control para la planta completa objeto de estudio

\section{REFERENCIAS}

[1] StePhanopoulus, G. Chemical Process Control. Prentice Hall, 1983.

[2] GARRIDO, J. Diseño de sistemas de control multivariable por desacoplo con controladores PID. Tesis (Ph.D.) Madrid: Escuela Técnica Superior de Ingeniería Informática, 2012.

[3] VÁZQUEZ, F. y MORILLA, F. Tuning decentralized PID controllers for MIMO systems with decoupling. In 15th IFAC World Congress, pp. 2172-2178, 2002.

[4] WANG, Q. G., HUANG, B. y GUO, X. Autotuning of TITO decoupling controllers from step tests. ISA Transactions, 39, pp. 407-418, 2000.

[5] WANG, Q. G. Decoupling Control. Lecture Notes in Control and Information Sciences, 285. Springer-Verlag, 2003.

[6] VÁZQUEZ, F. [et al]. An iterative method for tuning decentralized PID controllers. In 14th IFAC World Congress, pp. 491-496, 1999.
[7] CORRIPIO, A.B. y SMITH, C.A. Control automático de procesos: Teoría y Practica. Noriega Editores, 2000.

[8] BRISTOL, E. H. On a New Measure of Interaction for Multivariable Process Control. IEEE Transactions on Automatic Control, 11, 133-134, 1966.

[9] SKOGESTAD, S. y HAVRE, K. The use of RGA and condition number as robustness measures. Computers \& Chemical Engineering, 20, S1005-S1010, 1996.

[10] MCAVOY, T. J. Interaction analysis: Principles and applications, North Carolina, Instrument Society of America, 1983.

[11] NORDFELDT, P. PID Control of TITO Systems. Licentiate Thesis. Department of Automatic Control. Lund Institute of Technology. ISRN LUTFD2/TFRT--3228--SE. ISSN 0280-5316, 2005.

[12] NIEDERLINSKI, A. A Heuristic Approachto to me Design of Linear multivariable Control Systems, Automatic Vol.. 7, No. 691, 1971.

[13] ÅSTRÖM, K. J. y HÄGGLUND, T. Automatic tuning of simple regulators with specifications on phase and amplitude margins. Automatica, 20, 645-651, 1984.

[14] HÄGGLUND, T. y ̊̊STRÖM, K. J. Revisiting the Ziegler-Nichols step response method for PID control. Journal of Process Control, 14, 635-650, 2004.

[15] MORILLA, F. y DORMIDO, S. Methodologies for the tuning of PID controllers in the frequency domain. PID'0O IFAC Workshop on Digital Control: Past, present and future of PID Control. Terrassa, Spain, 2000.

[16] OCHOA, S. [et al]. Plantwide optimizing control of a continuous bioethanol production process. Journal of Process Control, 20(9), 983-998, 2010.

[17] LUYBEN, M. and FLOUDAS, C.A. Analyzing the interaction of design and control-1. A multiobjective framework and application to binary distillation synthesis. Computers and Chemical Engineering, 18(10), 933-969, 1994.

[18] LUYBEN, M. and FLOUDAS, C.A. Analyzing the interaction of design and control-2. Reactor-separator-recycle system. Computers and Chemical Engineering, 18(10), 971993, 1994.

[19] LUYBEN, M.L., TYREUS, B.D. and LUYBEN, W.L. Plantwide control design procedure. AIChE Journal, 43(12), 3161-3174, 1997.

[20] LUYBEN, W.L., TYREUS, B.D. and LUYBEN, M.L. Plantwide process control. McGraw Hill, New York, USA, 1999.

[21] RICARDO, J. F. y BARRIOS, E. Control Multivariable Lineal con Desacoples en un Intercambiador de Calor. Revista Ingeniería, Investigación y Desarrollo, Vol. 17 (1), pp. 17-25, Sogamoso-Boyacá. Colombia ISSN Impreso 1900-771X, ISSN Online 2422-4324, 2017. 